\title{
Alteração do óxido nítrico na função cardiovascular pelo treinamento físico
}

\section{Nitric oxide alteration in the cardiovascular function by exercise training}

\author{
Arthur de Freitas Brandão ${ }^{1}$; Marli Cardoso Martins Pinge ${ }^{2}$
}

Resumo

\begin{abstract}
Dentre os mecanismos de controle da pressão arterial, o controle humoral realizado pelo óxido nítrico (NO) tem despertado grande interesse científico. Os efeitos cardiovasculares observados após o treinamento físico estão associados ao aumento da estrutura vascular, bradicardia de repouso e um efeito hipotensor após esforço. O NO, sintetizado em células endoteliais a partir do aminoácido Larginina com a ação da enzima óxido nítrico sintase (NOS), é considerado um potente vasodilatador. O NO possui três isoformas enzimáticas: a enzima nNOS atua nas células nervosas e a eNOS em células endoteliais (ambas cálcio dependentes) e a iNOS, é ativada por estímulos imunológicos e independente do íon cálcio. No sistema nervoso central (SNC), o NO possui importante papel no controle da pressão sanguínea via sistema nervoso simpático, pois inibe sua atividade e diminui seu tônus sobre os vasos. Além disso, o NO exerce influência na região ventrolateral rostral medular (RVLM), importante na modulação da função cardiovascular. Estudos que relacionam o treinamento físico e $\mathrm{NO}$ verificaram significativo aumento na vasodilatação após treinamento físico em populações especiais como: hipertensos, obesos, diabéticos e cardiopatas, quando comparadas com indivíduos normais. Nesse sentido, parece haver uma modulação do treinamento físico sobre a participação do NO no controle da PA, tanto em nível periférico como central. Novos estudos se fazem ainda necessários para melhor elucidar este fenômeno.

Palavras-chave: Óxido nítrico. Vasodilatação. Treinamento físico. Pressão arterial.
\end{abstract}

\begin{abstract}
Among the mechanisms of arterial pressure control, the humoral one carried out by the nitric oxide (NO) has aroused great scientific interest. The cardiovascular effects observed after the physical training are associated to an increase in the vascular structure, resting bradycardia and a post effort hypotension effect. The NO that is synthesized in endothelial cells from the L-arginine amino acid with the nitric oxide synthase (NOS) action is considered a powerful vaso-dilator. The NO has three enzymatic isoforms: the nNOS enzyme is present in the nervous cells, and the eNOS in the endothelial cells, both calciumdependent. On the other hand, the iNOS which is activated by immunological stimuli, is calciumindependent. In the central nervous system (CNS), the NO exerts an important role in the blood pressure control through sympathetic nervous system, inhibiting its activity and decreasing the vascular tonus. Moreover, the NO exerts an influence on the rostral ventrolateral medulla (RVLM), which is important in the modulation of the cardiovascular function. Studies that relate physical training and NO, noticed a significant increase in vasodilation after physical training in special population such as: hypertensives, obese, diabetic and cardiopath people, when compared with healthy individuals. In this sense, there seems to be a modulation of the physical training on the NO participation in the PA control, not only in the peripherical but also in the central level. New studies are still necessary to better elucidate this phenomenon.
\end{abstract}

Key words: Nitric oxide. Vasodilation. Physical training. Arterial pressure.

1 Graduado em Educação Física.

2 Docente do Departamento de Ciências Fisiológicas da Universidade Estadual de Londrina. Mestrado em Fisiologia pela UNICAMP. Doutorado em Farmacologia pela UNIFESP. Pós-Doutorado na University of Columbia-Missouri, MO, USA. CEP: 86051-990. FAX: 3371-4467. Fone: 3371-4307. E-mail: martinspinge@uel.br

Semina: Ciências Biológicas e da Saúde, Londrina, v. 28, n. 1, p. 53-68, jan./jun. 2007 


\section{Introdução}

Segundo a Organização Mundial da Saúde (OMS), 15 milhões de mortes por ano são devidas a doenças cardiovasculares, correspondendo a $30 \%$ do total mundial de óbitos. Dois terços dessas mortes decorrentes de doenças cardiovasculares ocorrem em países em desenvolvimento, entre os quais o Brasil, que apresenta um número de mortes por doenças cardiovasculares duas vezes maiores do que países desenvolvidos (LAURENTI; BUCHALLA, 2001). Dessa maneira, compreender os eventos cardiovasculares, os efeitos de um determinado estilo de vida e o uso de fármacos, contribui para que a ciência possa estabelecer novas perspectivas de cura dessas doenças.

A literatura estabelece que os principais mecanismos de controle da pressão arterial envolvem: os barroreceptores, os quimioreceptores, o sistema renina angiotensina e os mecanismos humorais de controle local (IRIGOYEN et al., 2001; GUYTON; HALL, 2002).

Dentro dos fatores humorais de controle da pressão arterial, cita-se o óxido nítrico (NO), definido como fator relaxante do endotélio por Furchgott e Zawadzki, em 1980, e caracterizado posteriormente como potente vasodilatador do endotélio (MAEDA et al., 2004). Essas pesquisas geraram grande expectativa de que este gás tivesse forte envolvimento nos processos patológicos da hipertensão arterial. Sabe-se hoje que, além de ser liberado no endotélio, o NO é encontrado também no sistema nervoso central (em regiões específicas nos terminais nervosos) e regula desde o relaxamento de esfíncteres no sistema gastrointestinal até a transmissão de estímulos em áreas como a memória ou o olfato (SEARS; ASHLEY; CASADEI, 2004). Uma terceira função não menos importante do NO é conhecida por meio de sua liberação por estímulos imunológicos (TATCHUM-TALOM et al., 2000), induzida por macrófagos em resposta ao estímulo inflamatório (CHANG; CHAN; CHAN, 2003).

Por ser um gás instável, lipo-solúvel, o NO é sintetizado em células endoteliais a partir do aminoácido L-arginina com ação da enzima produzida no endotélio, a óxido nítrico sintase (NOS) (TATCHUM-TALOM et al., 2000; GREEN et al., 2004). Praticamente todas as células humanas estudadas até agora têm a capacidade de produzir NO (WANG, 2005). Atualmente, três isoformas de NOS foram identificadas: a neuronal (nNOS) e a endotelial (eNOS) (ambas cálcio-dependentes e expressas em condições fisiológicas) e a terceira, a isoforma induzida (iNOS), ativada por estímulos imunológicos e independente do íon cálcio (TATCHUM-TALOM et al., 2000).

O papel do óxido nítrico na função cardiovascular tornou-se importante após a localização das isoformas eNOS e nNOS no tecido cardíaco e nervoso (SEARS; ASHLEY; CASADEI, 2004; DANSON; PATERSON, 2005), pois nele, o NO derivado do endotélio (eNOS) possui importante papel no controle do tônus vascular (WANG, 2005). O controle autonômico cardíaco feito pelo núcleo do trato solitário (NTS) tem o NO derivado da nNOS como um de seus neuromoduladores (DANSON; PATERSON, 2005). Já em estruturas superiores do sistema nervoso central (SNC), o NO parece possuir um importante papel no controle da pressão sanguínea via sistema nervoso simpático (KIMURA et al., 2005).

\section{Mecanismos de controle da pressão arterial}

A manutenção dos níveis pressóricos dentro de uma faixa de normalidade depende de variações do débito cardíaco, da resistência periférica ou de ambos. Diferentes mecanismos de controle estão envolvidos não só na manutenção, como na variação, momento a momento, da pressão arterial, atuando na regulação do calibre e da reatividade vascular, na distribuição de fluídos dentro e fora dos vasos e no débito cardíaco. O estudo dos mecanismos de controle da pressão arterial tem indicado grande número de substâncias e sistemas fisiológicos que interagem de maneira complexa e com redundância para garantir a pressão arterial (PA) em níveis adequados nas mais diversas situações (IRIGOYEN et al., 2001). 
A PA pode ser calculada pelo produto da resistência vascular periférica total e pelo débito cardíaco (LOLIO, 1990; GUYTON; HALL, 2002). Assim, dentre os fatores que modificam o débito cardíaco, deve-se mencionar as alterações do volume sanguíneo, da contratilidade do miocárdio e da frequiência cardíaca. Já o controle da resistência vascular periférica total, depende do complexo mecanismo de regulação da resistência das arteríolas; do sistema renina-angiotensinaaldosterona; dos baroceptores do seio carotídeo, do arco aórtico e do átrio direito e neurotransmissores como a epinefrina e a norepinefrina (OIGMAN apud LOLIO, 1990).

O fluxo de sangue para cada tecido do corpo é controlado pela necessidade do tecido. Quando os tecidos estão ativos, eles requerem fluxo sanguíneo muito maior, do que quando estão em repouso (GUYTON; HALL, 2002). A pressão arterial é o mecanismo controlador do fluxo sanguíneo e da perfusão sanguínea no tecido, portanto, é por meio da pressão sanguínea que os órgãos e tecidos recebem as quantidades necessárias de oxigênio e retiram os metabólitos resultantes da atividade celular (AIRES, 1999).

É considerado um consenso que a maioria das alterações cardiovasculares estão relacionadas à alteração da estrutura e da função cardiovascular, e, nesse contexto, situa-se a disfunção endotelial como um dos importantes fatores que podem contribuir para o aumento da resistência periférica levando a complicações vasculares (IRIGOYEN, et al. 2000). Nesse sentido, o conhecimento dos mecanismos responsáveis pela manutenção de níveis normais de pressão arterial torna-se importante, uma vez que as doenças cardiovasculares mantêm sua liderança como uma das maiores causas de mortalidade (FERREIRA et al., 2000).

Os mecanismos responsáveis pelo controle da pressão arterial envolvem desde ativação neural pelos pressorreceptores arteriais ou barorreceptores, receptores cardiopulmonares, quimioreceptores arteriais até a ativação humoral como o sistema renina-angiotensina (IRIGOYEN et al., 2001). No contexto dessa revisão, vamos focar nos mecanismos humorais de controle e em especial o envolvimento do óxido nítrico.

\section{Mecanismos humorais no controle da pressão arterial}

O controle humoral da pressão arterial é exercido por vasodilatadores circulantes como cininas (bradicinina), polipeptídeo vasoativo intestinal, peptídeo atrial natriurético, adrenomedulina, e por vasoconstritores como, vasopressina, catecolaminas e angiotensina II (AMODEO et al., 1997).

A vasopressina liberada pela hipófise anterior participa do controle à curto prazo da pressão arterial, principalmente em situações de privação de água, depleção do volume sanguíneo e estados hipotensivos (AMODEO et al., 1997). Sua ação é tanto por via receptores renais $\mathrm{V}_{2}$, como por seu potente efeito vasoconstritor direto mediado por receptores $V_{1}$.

O aumento da pressão arterial e a elevação da frequiência cardíaca determinam a liberação do peptídeo natriurético atrial (ANP). Seu principal estímulo à liberação é o estiramento do átrio que pode ocorrer após expansão aguda da volemia (AMODEO et al., 1997). Para exercer seu efeito fisiológico, o ANP liga-se a receptores específicos em diferentes tecidos-alvo tais com o renal, o vascular e o adrenal estimulando a enzima guanilato ciclase com aumento na formação de GMPc via receptores tipo A (principalmente) e tipo B. Várias ações do ANP contribuem para a regulação da pressão arterial, incluindo seu potente efeito natriurético, facilitação do movimento dos fluídos para o espaço extravascular, vasodilatação e modulação da atividade do SRA-aldosterona (AMODEO, et al. 1997).

O sistema calicreína-cininas tem a bradicinina como um dos mais potentes vasodilatadores endotélio-dependentes conhecidos e existem várias evidências para síntese local de cininas no endotélio 
e células musculares lisas vasculares, no coração e nos rins. Em condições basais, as cininas parecem não influenciar a pressão arterial, uma vez que o bloqueio dos receptores $\mathrm{B}_{2}$ envolvidos na mediação dos efeitos cardiovasculares da bradicinina, não altera a pressão arterial. Com o bloqueio da ECA, no entanto, ocorre um aumento dos níveis endógenos de bradicinina, que parece tornar mais eficiente os efeitos crônicos dos inibidores da ECA em usuários hipertensos (AMODEO, et al. 1997).

A importância das substâncias com função parácrina (atuam sobre células vizinhas) produzidas especialmente no endotélio vascular para o controle da pressão arterial estão claramente estabelecidas (RIBEIRO, et al. 1992). Vasodilatadores como o óxido nítrico (NO) e prostaciclina, e vasoconstritores como a angiotensina II, endotelinas, radicais livres do oxigênio entre outros, desempenham papel crítico na manutenção da resistência periférica (AMODEO et al., 1997).

Em 1982, um ensaio biológico conduzido de maneira imprópria levou à descoberta de um fator constritor derivado do endotélio (EDCF). A intenção era testar a atividade biológica do meio de cultura proveniente das células endoteliais de aorta bovina sobre artérias coronárias isoladas de porcos. Os cientistas esperavam que as células endoteliais liberassem fatores relaxantes (EDRF) no meio de cultura e que este, por sua vez, relaxasse as preparações vasculares. Devido a uma extrema labilidade do EDRF, nenhuma atividade vasodilatadora foi encontrada quando da adição do meio de cultura às preparações, pois o doador (células endoteliais) e o aceptor (artérias coronárias) estavam em laboratórios diferentes. Ao invés de relaxamento, observou-se contração arterial lenta e de longa duração, e que não poderia ser atribuída a nenhum vasoconstritor conhecido. Em 1985, Hickey et al. demonstraram que o vasoconstritor presente na cultura de células endoteliais era um peptídeo de 21 aminoácidos, o qual foi denominado endotelina ou ET1 (AMODEO et al., 1997).

Nesta família dos peptídeos de 21 aminoácidos temos as endotelinas. Existem pelo menos 3 formas distintas de endotelina: ET-1, ET-2, ET-3, daas quais a mais potente vasoconstritora é a ET-1. O mecanismo de vasoconstrição pela endotelina inclui a ativação da fosfolipase $\mathrm{C}$, e levando a um aumento de inositoltrifosfato (IP3), com conseqüente elevação da concentração intracelular de cálcio. Além disso, a endotelina apresenta importante efeito potencializador de noradrenalina, outro vasoconstritor (AMODEO et al., 1997).

Dentre os vasodilatadores, o óxido nítrico (NO) tem recebido atenção especial a mais de uma década, principalmente após a demonstração da importância deste gás na manutenção do tônus vasodilatador. Sua síntese requer a oxidação do nitrogênio guanidino terminal da L-arginina dando origem ao metabólito intermediário. A formação do NO, em nível vascular, dá-se constitutivamente por meio de uma NO-sintase cálcio/calmodulina-dependente (AMODEO et al., 1997; TATCHUM-TALOM et al., 2000; GREEN et al., 2004). O NO produzido no endotélio promove vasodilatação por meio do aumento da formação do GMPc. Além disso, o NO apresenta importantes efeitos renais, cardíacos, na agregação plaquetária, modulação do ritmo de filtração glomerular (ROBERTS et al., 1999; MAEDA et al., 2004; WANG, 2005), entre outros aspectos.

\section{Formação e vias de produção do óxido nítrico}

Há muitos anos sabe-se que a resposta vasodilatadora produzida por vários agentes farmacológicos é mediada por uma substância produzida nas células endoteliais, substância essa denominada até pouco tempo de EDRF (endothelium derived relaxant factor) ou fator relaxante derivado do endotélio (GUYTON; HALL, 2002).

Estudos feitos por Furchgott e Zawadzki, em 1980, descreveram a vasodilatação em consequiência da ação de uma substância produzida no endotélio. Em 1987, foi sugerido que esse fator tinha características semelhantes às do NO (TATCHUM-TALOM et al., 2000).

O NO é um gás instável, lipo-solúvel, sintetizado em células endoteliais a partir do aminoácido L- 
arginina e com ação da enzima produzida no endotélio, a óxido nítrico sintase (NOS) (TATCHUM-TALOM et al., 2000; GREEN et al., 2004). Além de ser um potente vasodilatador, o NO exerce importante papel no controle da agregação plaquetária, resistência e crescimento vascular (MAEDA et al., 2004).

Praticamente todas as células humanas estudadas até agora têm a capacidade de produzir NO. A sua síntese ocorre durante a transformação do aminoácido semi-essencial L-arginina em L-citrulina, em uma reação mediada pela NOS, com a presença de vários co-fatores como oxigênio, NADPH, cálcio (dependendo do tipo de NOS), (WANG, 2005). Além disso, a aceticolina (Ach) produz vasodilatação pela estimulação farmacológica de receptores muscarínicos na célula endotelial; junto com a bradicinina, são consideradas dilatadoras dependentes de endotélio: substâncias que estimulam a síntese de NO a partir da L-arginina na célula endotelial (GREEN et al., 2004).

Atualmente, três isoformas de NOS foram identificadas: neuronal (nNOS) e endotelial (eNOS), sendo ambas cálcio-dependentes e expressas em condições fisiológicas. Em contraste, a isoforma induzida (iNOS) é ativada por estímulos imunológicos e independente do íon cálcio (TATCHUM-TALOM et al. 2000). Estudos recentes sugerem a presença de uma isoforma mitocôndrial de NOS com características intermediárias entre constitutiva e induzida (GIULIVI et al., 1998; TATOYAN et al., 1998).

Ao ser sintetizado, o NO, por ser uma molécula de pequena dimensão e altamente lipossolúvel, difunde-se por meio de células musculares lisas e estimula a enzima guanilato ciclase e a produção da 3,5 monofosfato de guanosina cíclico (GMPc). O GMPc aumenta a retomada de cálcio para dentro da célula por meio dos canais de cálcio. A proteína quinase dependente do GMPc, por sua vez, é ativada, levando à desfosforilação da miosina de cadeia leve, com conseqüente relaxamento muscular (ROBERTS et al., 1999).
O NO exerce diferentes ações, de acordo com o local de síntese, da quantidade produzida, tipo de isoenzima envolvida na sua liberação e microambiente onde vai atuar. Por exemplo, quando pequenas quantidades são liberadas nos terminais nervosos, o NO atua como neurotransmissor, regulando desde o relaxamento de esfíncteres no sistema gastrointestinal até a transmissão de estímulos no sistema nervoso central, em áreas como a memória ou o olfato. Quantidades similares, porém, se liberadas pelas células endoteliais, regulam o relaxamento da musculatura lisa adjacente, explicando a vasodilatação. As quantidades maiores produzidas em conseqüência a uma cadeia de estímulos envolvem o sistema enzimático da iNOS e podem ocasionar ou ampliar processos inflamatórios em vários órgãos (SEARS; ASHLEY; CASADEI, 2004). A iNOS é induzida por macrófagos em resposta ao estímulo inflamatório (CHANG; CHAN; CHAN, 2003).

As células cardíacas também são capazes de expressar a enzima eNOS e nesse sentido, a formação de NO nos miócitos tem uma função específica que é amplamente estudada. Aponta-se que o NO exerce função no controle da freqüência e contração cardíaca e protegendo os miócitos de morte isquêmica. Estudos mais recentes verificaram a presença de nNOS nos estoques de $\mathrm{Ca}^{2+}$ das células cardíacas, no nervo vago, na inervação simpática e nas regiões do controle autonômico sugerindo assim que esta isoforma tenha um papel importante ainda não demonstrado no controle e excitabilidade cardíaca (SEARS; ASHLEY; CASADEI, 2004).

O NO produzido pelo endotélio vascular regula o fluxo sanguíneo e mantém saudável a musculatura lisa dos vasos sanguíneos. A lesão do endotélio vascular prejudica a vasodilatação do vaso, e esta lesão pode ser associada com fatores de riscos para doenças vasculares, como na obstrução coronariana, lesão cardíaca crônica, e diabetes tipo 2 (MAIORANA et al., 2001). 


\section{O papel do óxido nítrico na função cardiovascular}

A identificação de inibidores competitivos da NOS, análogos da L-arginina tais como $\mathrm{N}^{\mathrm{G}_{-}}$ monometilarginina (L-NMMA) e nitroargininametiléster (L-NAME) ou $\mathrm{N}^{\mathrm{G}}$-nitro-Larginina metíl éster, possibilitou a investigação da relevância do $\mathrm{NO}$ em processos biológicos (AMODEO et al., 1997). O acúmulo de um inibidor endógeno da síntese de NO, a dimetilarginina assimétrica (ADMA), em insuficiência renal crônica, sugere que substâncias produzidas endogenamente podem regular a atividade da NOS local e sistemicamente (VALLANCE apud AMODEO et al., 1997).

No tecido cardíaco encontramos a enzima endotelial do NO (eNOS) e a neuronal (nNOS) (SEARS; ASHLEY; CASADEI, 2004; DANSON; PATERSON, 2005), ambas são constitutivas e dependentes de $\mathrm{Ca}^{+}$. Além dessas iso-formas, a (iNOS), iso-forma independente de $\mathrm{Ca}^{+}$, é expressa quando as células cardíacas são expostas a citocinas pró-inflamatórias (CSONT et al., 2005).

O NO derivado do endotélio (via eNOS), possui importante papel no controle do tônus vascular (WANG, 2005). O NO produzido pelas células endoteliais tem um potencial efeito vasodilatador (MAEDA et al., 2004).

Outro papel do NO gerado pela célula endotelial (eNOS) é a inibição da agregação e adesão plaquetária, por mecanismo dependente de GMPc, e trata-se de uma função sinégica com $\mathrm{PGI}_{2}$ (prostagladinas), que inibem a agregação plaquetária por aumento na concentração de AMPc (AMODEO et al., 1997). Estudos de Radomski e Moncada (1990) demonstraram que plaquetas também são capazes de gerar NO, o qual atua como mecanismo de retroalimentação negativa para inibir a ativação plaquetária. Portanto, a agregação plaquetária in vivo pode ser regulada por $\mathrm{NO}$ e $\mathrm{PGI}_{2}$ liberados pelo endotélio, bem como por NO liberado pelas plaquetas (MAEDA et al., 2004).
No controle autonômico central cardíaco, os impulsos aferentes vindos das terminações dos barorreceptores são integrados no sistema nervoso central, em especial no bulbo. O núcleo do trato solitário (NTS) recebe as informações das aferências dos barorreceptores via nervo glossofaríngeo e as transfere via núcleo ambíguo. Essas informações podem ser realizadas pelo $\mathrm{NO}$, devido ao fato de ter sido encontrado nNOS nos neurônios do NTS (LIN apud SEARS; ASHLEY; CASADEI, 2004) e no núcleo ambíguo (DUN apud SEARS; ASHLEY; CASADEI, 2004).

No sistema nervoso central, o NO é sintetizado e distribuído regionalmente pelos neurônios por meio da nNOS. No sistema vascular ocorre a síntese pela eNOS, embora esta ainda possa ser encontrada nos neurônios. O controle autonômico cardíaco feito pelo núcleo do trato solitário (NTS) tem o NO como um de seus neurotransmissores por meio de integrações aferentes originadas dos barorreceptores e por vias descendentes do cerebelo (DANSON; PATERSON, 2005).

O NO no sistema nervoso central (SNC), como no núcleo paraventricular do hipotálamo $(\mathrm{PVN})$ por exemplo, possui importante papel no controle da pressão sanguínea via sistema nervoso simpático. Em geral, no PVN o NO inibe a atividade do nervo simpático, diminuindo seu tônus sobre os vasos e reduzindo assim, a pressão sanguínea (PATEL et al., 2001).

Além de atuar no NTS, o NO liberado no sistema nervoso central por meio da nNOS exerce influência também na região do RVLM (bulbo ventrolateral rostral), região considerada fundamental para o tônus vasomotor (GUERTZENSTEIN; SILVER, 1974), por controlar elementos da função cardíaca (PATEL; LI; HIROOKA, 2001). A função cardiovascular é controlada por meio das eferências do RVLM sobre os neurônios pré-glanglionares simpáticos (DAMPNEY, 1994).

De fato, o NO parece desempenhar um papel funcional nos neurônios do RVLM, a partir do momento em que foi encontrado a NO sintase nesta região (IADECOLA et al., 1993; HIROOKA; 
POLSON; DAMPNEY, 1996). Alguns pesquisadores identificaram aumento da pressão arterial após micro-injeção de doadores de NO no RVLM (HIROOKA; POLSON; DAMPNEY, 1996; MARTINS-PINGE; BARALDI-PASSY; LOPES, 1997), no entanto outros pesquisadores encontraram queda da pressão arterial (SHAPOVAL; SAGACH; POBEGAILO, 1991; ZANZINGER; CZACHURSKI; SELLER, 1995; TSENG et al., 1996; KAGIYAMA et al., 1997). No estudo de Morimoto et al., (2000), foram usadas diferentes doses de doadores do $\mathrm{NO}$ ou precursores de $\mathrm{NO}$ e nele os autores demonstraram que pequenas doses de NO endógeno no RVLM causaram aumento na pressão arterial, mas com altas doses de NO verificou-se queda na pressão arterial. Outro estudo realizado por Chan, Wang e Chan (2001) mostrou que o RNAm das enzimas (iNOS) e (nNOS) estão presentes no RVLM em condições fisiológicas, e ambas são ativas em nível de expressão funcional e síntese molecular. Em um recente trabalho, foi observado que em animais acordados a iNOS e a nNOS estão tonicamente ativas no RVLM e ambas modulam a neurotransmissão glutamatérgica (MARTINS-PINGE et al., 2007). No estudo de Kimura et al. (2005), os resultados sugerem que expressão em excesso da iNOS no RVLM causa resposta hipertensora via ativação do sistema nervoso simpático, provavelmente atribuída ao aumento no estresse oxidativo.

Os dados da literatura em relação ao papel do NO no sistema nervoso central ainda permanecem contraditórios. Provavelmente isso se deva as várias metodologias utilizadas, espécies animais diferentes, drogas e concentrações diversas.

Tem-se assumido que qualquer efeito na contratilidade cardíaca, gerado pelo NO era resultado da NOS endotelial (eNOS). Com o avanço da tecnologia, hoje é possível desenvolver uma cepa de ratos knock-out (ratos que não produzem uma determinada enzima), específica para as iso-formas do NO e isso colaborou muito para o desenvolvimento dos estudos do papel do NO (SEARS; ASHLEY;
CASADEI, 2004). Ainda assim, fortes evidências sugerem que a estimulação do NO produzido no endotélio (eNOS) é capaz de modular a função do ventrículo esquerdo, em particular a função diastólica (CARTHY apud SEARS; ASHLEY; CASADEI, 2004). No entanto, em estudo com animais in vivo e in vitro knock-out para eNOS (GYURKO et al., 2000), foi mensurada e comparada a contratilidade dos miócitos, mas não foi observada diferença entre os knock-out e os animais do grupo controle, corroborando a idéia da eNOS ter pouco ou nenhum efeito na contratilidade cardíaca basal.

Estudos realizados por Shah e MacCarthy (2000) demonstraram que os efeitos do NO no relaxamento basal do ventrículo esquerdo são facilmente demonstrados, porém, em ratos knock-out para eNOS, o ventrículo esquerdo mostrou-se normal ou com aumento da função diastólica durante a estimulação $\beta$-adrenérgica. Os autores atribuíram estes achados a um aumento compensatório na expressão do peptídeo natriurético atrial e uma outra alternativa pode estar associada ao papel da nNOS no controle da função cardíaca ainda não identificado.

O NO produzido no miocárdio a partir da eNOS pode exercer importante papel no controle da função cardíaca, quando a enzima é ativada por estímulos específicos Por exemplo, quando ocorre aumento da elasticidade do tecido cardíaco ele se expande mais que o esperado. Essa expansão dos miócitos causa aumento do influxo de $\mathrm{Ca}^{2+}$ mediado pela produção do NO (SEARS; ASHLEY; CASADEI, 2004).

Em contrapartida, um estudo realizado com ratos knockout para a nNOS, sugere que a falta do estimulo da nNOS no ventrículo cardíaco pode inibir a contratilidade do miocárdio inibindo canais de cálcio do tipo-L (SEARS apud DANSON; PATERSON, 2005). Sugerem, que o efeito da nNOS na contratilidade do miocárdio sobre o sinal $\beta$-adrenérgico pode estar associado a concentração usada para os $\beta$-agonistas, adicionando a idéia de que, mais estudos são necessários para se definir o verdadeiro papel da enzima nNOS sobre a contratilidade cardíaca. 
Outro dado importante a respeito do papel da enzima neuronal em diferentes animais de experimentação é o fato de a inibição seletiva da nNOS causar redução no estímulo vagal e bradicardia no furão e no cachorro in vivo (COLON; MARKOS apud SEARS; ASHLEY; CASADEI, 2004), e o mesmo foi observado em preparações atriais isoladas de ratos. Este fenômeno pode ser explicado pela modesta contribuição da nNOS na resposta vagal (CHOATE apud SEARS; ASHLEY; CASADEI, 2004).

Quanto à isoforma enzimática iNOS, sabe-se que sua produção é induzida pelo processo próinflamatório de citocinas como: interleucina- $1 \beta$, fator de necrose tumoral e interferon- $\gamma$. Tem sido sugerido que o NO da iNOS exerce importante papel neste processo inflamatório: a inibição de sua produção em corações isolados previne a perda contrátil do tecido cardíaco (CSONT et al., 2005). Dessa maneira, vários estudos têm demonstrado importante papel do NO a partir da iNOS em diferentes situações cardiopatológicas (BOLI apud CSONT et al., 2005).

\section{Adaptações cardiovasculares ao exercício físico}

O exercício físico caracteriza-se por uma situação que retira o organismo de sua homeostase, pois implica no aumento instantâneo da demanda energética da musculatura exercitada e, conseqüentemente, do organismo como um todo. Assim, para suprir a nova demanda metabólica, várias adaptações fisiológicas são necessárias e, dentre elas, as referentes à função cardiovascular durante o exercício físico (BRUM, et al. 2004).

Já é de conhecimento que o exercício físico promove aumento da estrutura vascular, por exemplo, em estudos feitos a partir de autópsia e angiografia de artérias coronárias de atletas, foi encontrado um diâmetro de coronárias maior do que o comparado a indivíduos sedentários (PELLICIA, et al. 1990). No entanto, as respostas cardiovasculares dependem das características do exercício executado, ou seja, o tipo, a intensidade, a duração e a massa muscular envolvida (WILMORE; COSTILL, 2003).
Em relação ao tipo de exercício, podemos caracterizar dois tipos principais: exercícios dinâmicos ou isotônicos e exercícios estáticos ou isométricos, e cada um deles implica respostas cardiovasculares distintas (FORJAZ; TINUCCI, 2000).

Nos exercícios estáticos, observa-se um aumento da freqüência cardíaca, com manutenção ou até redução do volume sistólico e pequeno acréscimo do débito cardíaco. Em compensação, observa-se aumento da resistência vascular periférica, que resulta na elevação exacerbada da pressão arterial (WILMORE; COSTILL, 2003). Esses efeitos ocorrem porque a contração muscular mantida durante a contração isométrica (estática) promove obstrução mecânica do fluxo sangüíneo muscular, o que faz com que os metabólitos produzidos durante a contração se acumulem, ativando os quimiorreceptores musculares, que promovem aumento expressivo da atividade nervosa simpática resultando no aumento da pressão arterial (BRUM, et al. 2004).

Por outro lado, nos exercícios dinâmicos, as contrações são seguidas de movimentos articulares. Desse modo, não existe obstrução mecânica do fluxo sangüíneo, e nesse tipo de exercício, também se observa aumento da atividade nervosa simpática, que é desencadeado pela ativação do comando central, mecanorreceptores musculares e, dependendo da intensidade do exercício, metaborreceptores musculares (BRUM et al., 2004). Em resposta a esse aumento da atividade simpática, observa-se aumento da freqüência cardíaca, do volume sistólico e do débito cardíaco. Além disso, outros metabólitos musculares promovem a vasodilatação na musculatura ativa, promovendo diminuição da resistência periférica total (FORJAZ; TINUCCI, 2000).

Essas respostas são tanto maiores quanto maior for a intensidade do exercício, mas não se alteram com a duração do exercício, caso ele seja realizado numa intensidade inferior ao limiar anaeróbico (McARDLE, 2003). Além disso, quanto maior a massa muscular exercitada de forma dinâmica, maior 
é o aumento da frequiência cardíaca, porém menor é o aumento da pressão arterial (FORJAZ et al., 1998).

Dentre os principais parâmetros cardiovasculares que sofrem as adaptações ao treinamento físico estão a frequiência cardíaca e a pressão arterial, e um dos principais efeitos do treinamento físico é a diminuição da freqüência cardíaca em repouso (MEDEIROS, et al. 2000). Desse modo, a bradicardia de repouso tem sido considerada como um eficiente marcador do efeito do treinamento físico aeróbio (BRUM et al., 2000).

Em relação ao exercício aeróbio, a influência da duração desse exercício está bem demonstrada, apontando para o fato de que exercícios mais prolongados possuem efeitos hipotensores maiores e mais duradouros (FORJAZ et al., 1998b), principalmente após o treinamento físico com exercícios dinâmicos.

No que se refere ao exercício físico, outro fator de grande importância é sua relação com os efeitos cardiovasculares promovidos após o treinamento físico. Fenômenos que têm chamado a atenção são a "hipotensão pós-exercício" (BRUM et al., 2004), e o efeito anti-hipertensivo do exercício físico (HAMER, 2006). No trabalho de Hamer (2006), parece ficar clara a idéia de que a frequiência, intensidade e tempo de exercício são os fatores mais importantes para se obter queda na pressão arterial, principalmente em indivíduos hipertensos.

A hipotensão pós-exercício caracteriza-se pela redução da pressão arterial durante o período de recuperação, fazendo com que os valores pressóricos observados pós-exercício permaneçam inferiores àqueles medidos antes do exercício, ou mesmo àqueles medidos em um dia controle, sem a execução de exercícios. Para que a hipotensão tenha importância clínica, é necessário que ela perdure na maior parte das 24 horas subseqüente a finalização do exercício (BRUM et al., 2004; HAMER, 2006).

Estudos realizados no Laboratório de Fisiologia e Hemodinâmica da Atividade Motora da Escola de Educação Física e Esporte da Universidade de São
Paulo verificaram que em indivíduos normotensos, a execução de uma única sessão de 45 minutos de exercício em cicloergômetro em $50 \%$ do $\mathrm{VO}_{2}$ máximo reduz a pressão arterial sistólica/diastólica em torno de -7/-4 mmHg (BRUM et al., 2004).

Além disso, na população de normotensos, essa redução perdura por um período prolongado pósexercício, visto que a média da pressão arterial nas 24 horas pós-exercício estava diminuída. Entretanto, na população idosa normotensa, observou-se que uma sessão de exercício similar não promove redução da pressão arterial após sua execução. Por outro lado, em hipertensos, tanto jovens (SANTAELLA, 2003) quanto idosos (RONDON et al., 2002), a queda da pressão é mais evidente.

Outra confirmação de que o exercício físico causa bradicardia de repouso fo encontrado no estudo experimental de Medeiros, et al. (2000), realizado com ratos Wistar normotensos ( $\mathrm{n}=16)$, submetidos a treinamento físico com natação durante 8 semanas, cinco dias por semana, 60 minutos por dia. Houve, na freqüência cardíaca de repouso, uma diminuição de $10 \%$ ou 31 batimentos por minuto na freqüência cardíaca dos ratos treinados em relação aos sedentários, a partir da medida da pressão arterial indireta obtida por meio de esfigmomanômetro de cauda. Também foi verificado uma diminuição de $18 \%$ ou 66 batimentos por minuto a partir do pulso de pressão arterial (cânula intra-arterial) e uma diminuição de $8 \%$ ou 25 batimentos por minuto nos ratos treinados, comparados aos ratos sedentários, a partir do eletrocardiograma. Semelhantes resultados na freqüência cardíaca foram obtidos com o treinamento de natação por um período de tempo mais curto (4 semanas), em ratos Wistar normotensos com medida da PA de forma direta (MARTINSPINGE et al.,2005).

Em um outro estudo experimental com treinamento de natação para ratos, verificou-se aumento da atividade vagal e indução de hipertrofia cardíaca após treinamento de 8 semanas (MEDEIROS, et al. 2004). Após a sexta semana de 
treinamento, houve uma significativa diminuição da freqüência cardíaca do grupo treinado, e isso caracteriza uma bradicardia de repouso. $\mathrm{O}$ mesmo resultado dos estudos anteriores o qual é característico de exercícios aeróbicos.

O exercício físico promove também importante papel nos canais de cálcio e potássio na artéria mesentérica e artéria aorta. No estudo de Chen e colaboradores (2001), ratos foram submetidos a caminhadas na esteira por uma hora/dia, cinco dias por semana, durante 8 semanas à aproximadamente $65 \%$ da capacidade cardiovascular máxima, após breve período de adaptação. Os resultados apresentados mostram que o treinamento provocou aumento na condução elétrica nos canais de cálcio $\left(\mathrm{Ca}^{+}\right)$e potássio $\left(\mathrm{K}^{+}\right)$nas artérias analisadas, promovendo relaxamento das artérias pela ação da acetilcolina (Ach) e aumento plasmático de nitrato (metabólito do NO), o que sugere aumento na formação de óxido nítrico na artéria mesentérica e artéria aorta nos ratos que foram submetidos ao programa de exercícios.

Os efeitos hipotensores do exercício físico são considerados um consenso na literatura atual, no entanto, esse efeito é mais pronunciado em indivíduos hipertensos. Com efeito, a maioria dos estudos realizados em normotensos não mostrou modificação da pressão arterial ou, então, houve reduções de pequena magnitude, tanto na pressão arterial de consultório como na monitorização ambulatorial da pressão arterial de 24 horas (SILVA, et al. 1997). Entretanto, somente o treinamento físico realizado em intensidade leve a moderada, correspondente a $55 \%$ do $\mathrm{VO}_{2}$ máximo, atenuou a hipertensão arterial de ratos com hipertensão severa quando comparados a ratos sedentários e treinados em $85 \%$ do $\mathrm{VO}_{2}$ máximo. O mecanismo hemodinâmico envolvido na atenuação da hipertensão nesses animais foi a redução do débito cardíaco associada a bradicardia de repouso e redução do tônus simpático cardíaco (GRAHAM, et al. 2004).

Nesse sentido, uma das disfunções neurovegetativas associadas à hipertensão arterial é a diminuição da sensibilidade dos reflexos cardiovasculares, tais como, o reflexo pressorreceptor e cardiopulmonar, que são importantes para a regulação momento-a-momento da pressão arterial. Esta seria, ademais, uma das causas do alto índice de mortalidade por doenças cardiovasculares, especialmente na insuficiência cardíaca (BRUM, et al. 2004).

Está bem estabelecido na literatura que a prática de exercício físico, principalmente os aeróbicos que envolvem a participação de grandes porções musculares (caminhada, corrida, natação, etc) (WILMORE; COSTILL, 2003), é capaz de modular a pressão arterial nos níveis normais (IRIGOYEN, et al. 2003; BRUM et al., 2004) promovendo efeito hipotensor logo após o esforço (HAMER, 2006). Acredita-se que o óxido nítrico (NO) esteja envolvido nessa modulação.

\section{Alterações na disponibilidade do óxido nítrico após treinamento físico}

Segundo GREEN et al., (2004), são bem divergentes os resultados das pesquisas com a participação do óxido nítrico e seu efeito no treinamento físico.

Por exemplo, em jovens saudáveis não foram encontradas alterações no efeito do óxido nítrico, nem aumento na síntese do NO, para exercícios localizados no ante-braço, depois de quatro semanas de treinamento 3 vezes por semana (GREEN et al. 1994, FRANK apud GREEN, et al., 2004). Já em pacientes com insuficiência cardíaca, 30 minutos de exercícios localizados para ante-braço de 4 a 6 vezes por semana, trouxeram um aumento da síntese de NO e aumento da atividade da acetilcolina após o treinamento (BANK apud GREEN et al., 2004).

Exercícios que envolvem a participação de grandes porções musculares, os resultados também são divergentes. Foi observado, em recrutas saudáveis do Exército, aumento da síntese de NO após o seguinte treinamento: corridas de 3 milhas por dia 
mais sessões de treinamento de força, por 10 semanas, o que caracteriza um alto volume de treinamento físico (CLARKSON apud GREEN et al., 2004).

Homens jovens apresentaram queda na vasodilatação mediada por acetilcolina analisada após treinamento, o qual constou de 4 sessões por semana de corrida a $70 \%-80 \%$ do $\mathrm{VO}_{2 \text { máx. }}$ subestimado ou seja, um treinamento considerado de alta intensidade por uma hora de duração em cada sessão. Segundo o autor, estes efeitos podem ter ocorrido devido à queda na concentração de antioxidantes (BERGHOLM apud GREEN et al., 2004). Dessa forma, parece evidente que a intensidade do exercício determina o papel do NO no efeito vasodilatador.

Já indivíduos normais de meia idade não apresentaram alterações na vasodilatação mediada pela concentração de acetilcolina, nem no aminoácido essencial para formação do NO, L-arginina. Isso ocorreu após terem realizado treinamento de circuito de resistência em aparelhos de musculação com 7 exercícios gerais para membros inferiores, tronco, e membro superiores, todos em moderada intensidade, 3 vezes por semana durante 8 semanas (MAIORANA et al., 2001). Neste trabalho, ficou caracterizado que, exercícios isolados não apresentam melhoras significativas nas respostas cardiovasculares ao treinamento físico.

Diferentemente, para indivíduos de meia idade, porém sedentários (DE SOUZA et al. 2000, apud GREEN, et al. 2004) ou hipertensos (HIGASHI et al. 1999a apud GREEN et al. 2004), 30 minutos de caminhada de 5 a 7 vezes por semana durante 3 meses, representaram um significativo aumento da síntese de NO e de seu efeito vasodilatador. Esse fato sugere os exercícios aeróbicos envolvem grandes leitos musculares, e intensa participação do sistema cárdiopulmonar na execução das atividades, de modo que com eles obtêm-se efeitos significativos nas respostas cardiorespiratórias.

Nos estudos encontrados com indivíduos obesos envolvidos em exercícios de caminhada, circuito de treinamento ou praticando esportes, houve um aumento da produção de NO, vasodilatação após o exercício e até melhora na curva dose-resposta para acetilcolina, (SCIACQUA et al. 2003, WATTS, et al. 2004a, 2004b, apud GREEN, et al. 2004).

Da mesma maneira, estudos com diabéticos tipo 1 envolvidos durante 4 meses em exercícios com bicicleta ergométrica, por 1 hora 3 sessões por semana, apresentaram aumento significativo da vasodilatação mediada pelo NO, (FUCHSJAGERMAYRL, et al. 2002, apud GREEN, et al. 2004).

Estes estudos indicam que o exercício físico envolvendo os membros inferiores ou maiores porções musculares, melhoram a função vascular mediada pela vasodilatação promovida pelo NO, diferentemente do que ocorre com exercícios exclusivos para membros superiores (MAIORANA et al., 2000, LINKE apud GREEN et al., 2004).

O aumento na vasodilatação dependente do endotélio vascular causada pelo treinamento da musculatura tem uma forte correlação com as alterações da capacidade funcional e um pequeno aumento do débito cardíaco (HAMBRECHT apud GREEN et al., 2004).

Em resumo, a melhora da vasodilatação mediada pelo NO em pacientes com problemas crônicos de: hipertensão, diabetes e hipercolesterolemia, está mais associada à melhora da capacidade funcional dos pacientes do que aos aumentos da produção do NO (GREEN et al., 2004).

Outro estudo com 15 mulheres idosas (59-69 anos), submetidas a um programa de exercício físico em ciclo ergômetro por 30 minutos/dia, 5 dias por semana, a $80 \%$ de seu limite respiratório individual, cerca de $50 \%$ do $\mathrm{VO}_{2 \text { máx. }}$, durante 3 meses, promoveu aumento significativo da produção basal de óxido nítrico e GMPc (MAEDA et al., 2004). Esse fenômeno pode estar relacionado ao efeito antihipertensivo e anti-colesterolêmico da produção endógena do NO no sistema vascular promovida pela prática de exercícios físicos periódicos. O significativo aumento na produção do NO, nesta 
situação, implica maior controle do tônus vascular e ajuda a prevenir a progressão de arteriosclerose. Dessa maneira, os autores sugerem que mulheres idosas pratiquem exercícios físicos diários com o objetivo de diminuir os riscos de acidentes cardiovasculares (MAEDA et al., 2004).

O exercício físico parece promover a liberação e aumento da bio-disponibilidade de $\mathrm{NO}$ em vários tecidos cardíacos. Esse efeito parece ocorrer por dois mecanismos. No primeiro, ocorre um aumento da atividade da eNOS no endotélio vascular coronariano após exercício de esteira em ratos, devido ao estresse hemodinâmico provocado pelo sangue durante o exercício. Estes efeitos melhoram o relaxamento vascular e, posteriormente, a perfusão sanguínea no miocárdio. No segundo, verifica-se o aumento dos níveis de NO, em correlação ao aumento da nNOS após o exercício. Isso permite que as áreas de controle autonômico cardíaco tenham maior sensibilidade as alterações da pressão arterial e fluxo sanguíneo (DANSON; PATERSON, 2005).

\section{Conclusão}

O papel vascular do óxido nítrico com o treinamento físico é bastante divergente na literatura, contudo podemos concluir que o treinamento físico é capaz de alterar significativamente a biodisponibilidade, bem como os efeitos do NO no sistema cardiovascular.

Observamos que, em humanos, exercícios localizados, realizados por pequenos grupos musculares, não alteram a produção e o efeito do NO nos leitos vasculares. Entretanto em indivíduos normais e saudáveis, exercícios gerais, principalmente os exercícios aeróbicos como caminhada, corrida, natação, etc, são capazes de promover aumento na biosíntese de NO bem como aumento de seu efeito vasodilatador.

Apesar disso, quando o exercício realizado é de alta intensidade, os indivíduos apresentaram queda na vasodilatação mediada pelo $\mathrm{NO}$, e isso sugere uma intensidade adequada de treinamento físico que seja considerada ótima para que o efeito benéfico de vasodilatação pelo NO apareça.

Indivíduos sedentários, hipertensos, diabéticos e obesos apresentaram aumento no efeito vasodilatador mediado pelo $\mathrm{NO}$, quando envolvidos em qualquer tipo de treinamento físico, seja caminhada, exercício em bicicleta ergométrica, circuito de musculação ou praticando esportes. Parece claro, que para esta população, qualquer tipo de exercício trará algum tipo de benefício no controle de suas patologias.

Parece claro que o treinamento físico melhora a função vasodilatadora pelo NO de forma direta e indireta. Fatores convencionais que têm implicações deletérias na função vasodilatadora pelo NO tem se beneficiado pelo treinamento físico (GREEN et al., 2004). Nesse processo, o treinamento físico mais prolongado apresenta remodelagem vascular com aumento do diâmetro do vaso, permitindo nessa fase que a bioatividade do NO retorne aos níveis prétreinamento. Essa hipótese é baseada em estudos onde se observaram que o aumento da vasodilatação pelo NO ocorre em treinamento físico de curto à médio prazo, e em treinamentos de longo prazo, associados com remodelação vascular, não tem sido observado aumento na função endotelial (GREEN et al., 2004).

$\mathrm{O}$ esclarecimento dos mecanismos celulares e o tipo de treinamento físico mais adequado para promover aumento da vasodilatação vascular mediada pelo NO, bem como, a descoberta de novos fármacos que controlem ou aumentem a disponibilidade de NO, pode contribuir para a diminuição dos níveis de hipertensão arterial, bem como, diabetes e obesidade, e promover a melhora da qualidade de vida dos pacientes. 


\section{Referências}

AIRES, M. M. Fisiologia. 2.ed. Rio de Janeiro: Guanabara Koogan, 1999.

AMODEO, C.; LIMA, E. G.; VASQUEZ, E. C. Hipertensão arterial, São Paulo: Saraiva; Sarvier, 1997.

BRUM, P. C.; SILVA, G. J. J.; MOREIRA, E. D.; IDA, F.; NEGRAO, C. E.; KRIEGER, E. M. Exercise training increases baroreceptor gain sensitivity in normal and hypertensive rats. Hypertension, Dallas, v.36, n.6, p.10181022, dec. 2000.

BRUM, P. C.; FORJAZ, C. L. M; TINUCCI, T.; NEGRÃO, C. E. Adaptações agudas e crônicas do exercício físico no sistema cardiovascular. Revista Paulista de Educação Física, São Paulo. v.18, n.esp., p.21-31, ago. 2004.

CHAN, J. Y. H.; WANG, S. H.; CHAN, S. H. H. Differential roles of iNOS and nNOS at rostral ventrolateral medulla during experimental endotoxemia in the rat. Shock, Philadelphia, v.15, p.65-72, 2001.

CHANG, A. Y. W.; CHAN, J. Y. H.; CHAN, S. H. H. Differential distribution of nitric oxide synthase isoforms in the rostral ventrolateral medulla of the rat. Journal of. Biomedical Science, Basel, v.10, n.3, p.285-291, may 2003.

CHEN, S. J.; WU, C. C.; YEN, M. H. Exercise training activates large-conductance calcium-activated $\mathrm{K}^{+}$ channels and enhances nitric oxide production in rat mesenteric artery and thoracic aorta. Journal of Biomedical Science, Basel, v.8, n.3, p.248-255, may 2001.

CSONT, T.; VIAPPIANI, S.; SAWICKA, J.; SLEE, S.; ALTAREJOS, J. Y.; HABERLE, I. B.; SCHULZ, R. The involvement of superoxide and iNOS-derived NO in cardiac dysfunction induced by pro-inflammatory cytokines. Journal of Molecular and Cellular Cardiology. London, v.39; n.5, p.833-840, nov. 2005.

DAMPNEY, R. A. Functional organization of central pathways regulating the cardiovascular system. Physiological Reviews, Baltimore, v.74, n.2, p.323-364, 1994

DANSON, E. J.; PATERSON, D. J. Cardiac neurobiology of nitric oxide synthaes. Annals New York Academy of Sciences, New York, v.1047, n.1, p.183-196, jun. 2005.

FERREIRA, S. R. G.; ZANELLA, M. T.; Epidemiologia da hipertensão arterial associada à obesidade Revista Brasileira Hipertensão, São Paulo, v.7, n.2, p.128-135.abr./ jun.2000.

FERRARIO, C. M.; MILSTED, A.; SANTOS, R. A. S. Renin, converting enzyne and angiotensin peptides:review. In: KEANE, W. F. (Ed.). Afocus on the clinical effects of a long-acting ACE inihibitor/Hypertension. New York: Raven Press, 1990. p.1-19.
FORJAZ, C. L. M.; TINUCCI, T. A medida da pressão arterial no exercício. Revista Brasileira de Hipertensão, São Paulo, v.7, n.1, p.79-87, 2000.

FORJAZ, C. L. M.; MATSUDAIRA, Y.; RODRIGUES, F.B.; NUNES, N.; NEGRÃO, C.E. Post-exercise changes in blood pressure, heart rate and rate pressure product at different exercise intensities in normotensive humans. Brazilian Journal Medicine Biological Research, Ribeirão Preto, v.31, n.10, p.1247-55, 1998.

FORJAZ, C. L. M.; SANTAELLA, D. F.; REZENDE, L. O.; BARRETTO, A. C. P.; NEGRÃO, C. E. A duração do exercício determina a magnitude e a duração da hipotensão pós-exercício. Arquivos Brasileiros de Cardiologia, São Paulo, v.70, n.2, p.99-104, 1998b.

GIULIVI, C.; PODEROSO, J. J.; BOVERIS, A. Production of nitric oxide by the mitochondria. The Journal of Biological Chemistry, Bethesda, v.273: n.18, p.1103811043, may 1998.

GRAHAM, D. A.; RUSH, J. W. Exercise training improves aortic endothelium-dependent vasorelaxation and determinants of nitric oxide bioavailability in spontaneously hypertensive rats. Journal of Applied Physiology, Bethesda, v.96, n.6, p.2088-2096, jun.2004.

GREEN, D.J.; CABLE, N.T.; FOX, C.; RANKIN, J.M.; TAYLOR, R.R. Modification of forearm resistance vessels by exercise training in young men. Journal of Applied Physiology, v.77(4), 1829-1833, 1994.

GREEN, D. J.; MAIORANA, A.; O'DRISCOLL G.; TAYLOR, R. Effect of exercise training on endotheliumderived nitric oxide function in humans. The Journal of. Physiology, Cambridge, v.561, n.1; p.1-25, nov.2004.

GUERTZENSTEIN, P. G; SILVER, A. Fall in blood pressure produced from discrete regions of the ventral surface of the medulla by glycine and lesions. The Journal of.Physiology, Cambridge, v.242, p.489-503. 1974.

GUYTON, A.; HALL, J. Tratado de fisiologia médica, 10.ed. São Paulo: Guanabara Koogan, 2002.

GYURKO, R.; KUHLENCORDT, P.; FISHMAN, M. C.; HUANG, P. L. Modulation of mouse cardiac function in vivo by eNOS and ANP. American Journal of Physiology: Heart and Circulatory Physiology, Bethesda, v.278, H971H981, mar. 2000.

HAMER, M. The anti-hypertensive effects of exercise: integrating acute and chronic mechanisms. Sports Medicine, Auckland, v.36, n.2, p.109-16. 2006.

HIROOKA, Y.; POLSON, J. W.; DAMPNEY, R. A. Pressor and sympathoexcitatory effects of nitric oxide in the rostral ventrolateral medulla. Journal of Hypertension, London, v.14, p.1317-1324, 1996. 
IADECOLA, C.; FARIS, P. L.; HARTMAN, B. K.; XU, X. Localization of NADPH diaphorase in neurons of the rostral ventrolateral medulla: possible role of nitric oxide in central autonomic regulation and oxigen chemoreception. Brain Reearsh, Amsterdam,. v.603, n.1, p.173-179, 1993.

IRIGOYEN, M. C.; LACCHINI, S.; ANGELIS, K.; MICHELINI, L. Fisiopatologia da Hipertensão: O que avançamos? Revista da Sociedade de Cardiologia do Estado de São Paulo. São Paulo, v.13, n.1, p.20-45. jan./ fev. 2003.

IRIGOYEN, M. C., CONSOLIM-COLOMBO, F. M.; KRIEGER, E. M. Controle cardiovascular: regulação reflexa e papel do sistema nervoso simpático. Revista Brasileira de Hipertensão, São Paulo, v.8, p.55-62, 2001.

IRIGOYEN, M. C. Alterações funcionais do sistema cardiovascular durante o envelhecimento. In: JECKELNETO, E. A.; CRUZ, I. B. Aspectos biológicos e geriátricos do envelhecimento II. Porto Alegre: EDIPUCRS; 2000. p.337-70.

IRIGOYEN, M. C.; MOREIRA, R. D.; MOREIRA, E. D.; KRIEGER, E. M. High-renin hypertension depresse the baroreflex control of heart rate and sympathetic activity. In: CIRIELLO, J. Central Neural Mechanisms in Blood Pressure regulation. Local: Spring-Verlag, 1991. p.252-262.

KAGIYAMA, S.; TSUCHIHASHI, T.; ABE, I.; FUJISHIMA, M. Cardiovascular effects of nitric oxide in the rostral ventrolateral medulla of rats. Brain Research, Amsterdam, v.757, n.1, p.155-158, 1997.

KIMURA, Y.; HIROOKA, Y.; SAGARA, Y.; ITO, K.; KISHI, T.; SHIMOKAWA, H.; TAKESHITA, A.; SUNAGAWA, $\mathrm{K}$. Overexpression of inducible nitric oxide synthase in rostral ventrolateral medulla causes hypertension and sympathoexcitation via an increase in oxidative stress. Circulation Researsh, Baltimore, v.96, n.2, p.252-260, fev.2005.

LAURENTI, R.; BUCHALLA, C. M. Acabar com os mitos sobre as cardiopatias. Arquivo brasileiro de Cardiologia, Rio de Janeiro, v.76.n.2, p.99-104, 2001.

LOLIO, C. A. The epidemiology of arterial hypertension. Revista da Saúde Pública. São Paulo, v.24; n.5, p.425432. Oct. 1990.

MAEDA, S.; TANABE, T.; OTSUKI, T.; SUGAWARA, J.; IEMITSU, M.; MIYAUCHI, T.; KUNO, S.; AJISAKA, R.;MATSUDA, M. Moderate Regular Exercise Increases Basal Production of Nitric Oxide in Elderely Women. Hypertens Res. 27, n.12:947-953, dec.2004.

MAIORANA, A.; O'DRISCOLL, G.; DEMBO, L.; CHEETHAM, C.; GOODMAN, C.; TAYLOR, R.; GREEN, $D$. Effect of aerobic and resistance exercise training on vascular function in heart failure. American Journal of Physiology, 279(4), H1999-H2005, 2000.

MAIORANA, A.; O’DRISCOLL, G.; DEMBO, L.; GOODMAN, C.; TAYLOR, R.; GREEN, D. Exercise trainig, vascular function, and functional capacity in middle-aged subjects. Medicine \& Science in Sport \& Exercise, Baltimore, v.33, n.12, p.2022-2028, dec. 2001.

McARDLE, W. et al. Fisiologia do Exercício: Energia nutrição e Desempenho humano. 5.ed. Rio de Janeiro: Guanabara Koogan. 2003.

MARTINS-PINGE, M. C.; BARALDI-PASSY, I.; LOPES, O.U. Excitatory effects of nitric oxide within the rostral ventrolateral medulla of freely moving rats. Hypertension, Dallas, v.30, p.704-707, 1997.

MARTINS-PINGE, M.C.; BECKER, L. K, GARCIA, M. R. L.; ZOCCAL, D. B; VASCONCELLOS NETO, R.; BASSO, L. S.; SOUZA, H. C.; LOPES, O. U. Attenuated pressor responses to amino acids in the rostral ventrolateral medulla after swimming training in conscious rats. Autonomic Neuroscience., Amsterdam, v.122, n.1-2, p.2128, 2005.

MARTINS-PINGE, M. C.; GARCIA, M. R. L;ZOCCAL, D. B.; CRESTANI, C. C.; PINGE-FILHO, P. Differential influence of iNOS and nNOS inhibitors on rostral ventrolateral medullary mediated cardiovascular control in conscious rats. Autonomic Neuroscience. Amsterdam, v.131, n.1-2, p.65-69, 2007.

MEDEIROS, A. ; OLIVEIRA, E. M.; GIANOLLA, R.; CASARINI, D. E.; NEGRÃO, C. E.; BRUM, P. C.

Swimming training increases cardiac vagal activity and induces cardiac hypertrophy in rats. Brazilian Journal of Medical Biololoical Research Ribeirão Preto, v.37, n.12, p.1909-1917, dec.2004.

MEDEIROS, A.; GIANOLLA, R. M.; KALIL, L. M. P.; BACURAU, R. F. P.; ROSA, L. F. B. C.; NEGRÃO, C. E.; BRUM, P. C. Efeito do treinamento físico com natação sobre o sistema cardiovascular de ratos normotensos. Revista Paulista de Educação Física, São Paulo, v.14, n.1, p.7-15, jan./jun. 2000.

MONCADA, S. PALMER, R. M.; HIGGS, E. A. Nitirci oxide:physiology, pathophysiology and pharmacology. Pharmacological Review, v.43, n.2, p.109-142, Jun.1991.

MORIMOTO, S.; SASAKI, S.; MIKI, S.; KAWA, T.; NAKAMURA, K.; ITOH, H.; NAKATA, T.; TAKEDA, K.; NAKAGAWA, M.; FUSHIKI, S. Nitric oxide is an excitatory modulator in the rostral ventrolateral medulla in rats. American Journal Hypertension, New York, v.13, n.10, p.1125-1134. Oct.2000. 
OIGMAN, W. Bases hemodinâmicas da hipertensão arterial. Arquivos Brasileiros de Cardiologia, São Paulo,. v.49, p.303-8, 1987.

PATEL KP, LI YF, HIROOKA Y. Role of nitric oxide in central sympathetic outflow. Expimental Biology and Medicine, Maywood, v.226, n.9, p.814-824, 2001.

PELLICIA, A.; SPATARO A.; GRANATA M.; BIFFI, A,; CASELlI, G.; ALABISO, A. Coronary arteries in physiological hypertrophy: echocardiographic evidence of increased proximal size in elite athletes. International Journal of Sports Medicine, Stuttgard, v.11, p.120-126, 1990.

RIBEIRO, M. O.; ANTUNES, E.; DE NUCCI, G.; LOVISOLO, S. M.; ZATZ, R. Chronic inhibition of oxide synthesis. A new model of arterial hypertension. Hypertensionsion, Dallas, v.20, n.3, p.298-303, sep.1992.

ROBERTS, C. K.; BARNARD, R. J.; JASMAN, A.; BALON, T. W. Acute exercise increases nitric oxide synthase activity in skeletal muscle. American Physiological Society: Endocrinology and Metabolism: Rapid Communication, Bethesda, v.277, n.2, p.E390-E394, Aug.1999.

Acute exercise increases nitric oxide synthase activity in skeletal muscle. American Physiological Society Endocrinology and Metabolism:Rapid Communication. v.277, n.2, p.E390-E394, Aug.1999.

RONDON, M. U. P. B.; ALVES, M. J. N. N.; BRAGA, A M. F. B.; TEIXEIRA, U. T. O. N.; BARRETTO, A. C. P.; KRIEGER, E. M.; NEGRÃO, C. E. Postexercise blood pressure reduction in elderly hypertensive patients. Journal of the American College of Cardiology. New York, v.39, n.4, p.676-82, feb.2002.

SANTAELLA, D. F. Efeitos do relaxamento e do exercício físico nas respostas pressórica e autonômica pósintervenção em indivíduos normotensos e hipertensos. 2003. Dissertação (Mestrado em Fisiopatologia Experimental) - Faculdade de Medicina, Universidade de São Paulo, São Paulo.
SEARS, C. E.; ASHLEY, E. A.; CASADEI, B. Nitric oxide control of cardiac function: is neuronal nitric oxide synthase a key component? Philosophical Transactions Royal Society of London, London, v.359: p.1021-1044, 2004.

SHAH, A. M.; MAcCARTHY, P. A. Paracrine and autocrine effects of nitric oxide on myocardial function. Pharmacology Therapeutics, New York, v.86, n.1, p.4986. 2000.

SHAPOVAL, L. N.; SAGACH, V. F.; POBEGAILO, L. S. Nitric oxide influences ventrolateral medullary mechanisms of vasomotor control in the cat. Neuroscience Letters, Limerick, v.132: p.47-50, 1991.

SILVA, G. J. J.; BRUM, P. C.; NEGRÃO, C. E.; KRIEGER, E. M. Acute and chronic effects of exercise on baroreflexes in spontaneously hypertensive rats. Hypertension, Dallas, v.30, p.714-9, 1997.

TATCHUM-TALOM, R.; SCHULZ, R.; McNEILL, J. R.; KHADOUR; F. H. Upregulation of neuronal nitric oxide synthase in skeletal muscle by swim training. American Journal Physiology:Heart Circulation Physiology, Bethesda, 279(4): H1757-66. Oct.2000.

TSENG, C. J.; HUI-YA, L.; HUI-CHING, L.; LUO-PING, G.; CHE-SE, T.; MAO-HSIUNG, Y. Cardiovascular effects of nitric oxide in the brain stem nuclei of rats. Hypertension. Dallas, v.27, n.1, p.36-42, jan.1996.

WANG, J. S. Effects of exercise training and detraining on cutaneous microvascular function in man: the regulatory role of endothelium-dependent dilation in skin vasculature. European Journal Applied Physiology, Heidelberg, v.93: n.4, p.429-434, jan.2005.

WILMORE, J.H.; COSTILL, D.L. Physiology of Sport and Exercise. 2 ed. Champaign Ilinois, Human Kinetics. 2003

ZANZINGER, J.; CZACHURSKI, J.; SELLER, H. Inhibition of basal and reflex-mediated sympathetic activity in the RVLM by nitric oxide. American Journal of Physiology, Baltimore, v.268, p.R958-R962,1995. 
